\title{
Die Behandlung des Holocaust im Nürnberger Prozess gegen die Hauptkriegsverbrecher und die Wahrnehmung in der deutschen Nachkriegsöffentlichkeit
}

\section{Dietmar RiedI}

Kerngebiet: Zeitgeschichte

Eingereicht bei: assoz. Prof. Mag. Dr. Eva Pfanzelter (MA)

Eingereicht im: WS 2016/17

Rubrik: PS-Arbeit

\begin{abstract}
The treatment of the Holocaust in the Nuremberg trial against the main war criminals and the perception in the postwar German public

The following pro-seminar paper deals with the treatment of the Holocaust in the Nuremberg trial against the main war criminals of the national-socialist regime, its value in the framework of the trial and its influence on the judgement as well as its perception and legal overcoming in postwar Germany.
\end{abstract}

\section{Einleitung}

„Die verhafteten NS-Führer wegen ihrer Verbrechen vor Gericht zu stellen war einfach. Wollte man aber einen solchen Prozess führen, musste man sich auf ein rechtliches Minenfeld begeben, denn für das Internationale Militärtribunal (mit Richtern und Staatsanwälten der vier Besatzungsmächte), das 1945/46 ein Jahr lang in Nürnberg tagte, gab es weder einen Präzedenzfall noch eine gängige Rechtsprechung."

So beschreibt der englische Historiker lan Kershaw die Situation zu Beginn des Prozesses gegen die Hauptkriegsverbrecher des nationalsozialistischen Deutschland im November 1945.

Ian Kershaw, Höllensturz. Europa 1914 bis 1949, München 2016, S. 650-651. 
Tatsächlich endete mit der Urteilsverkündung am 30. September bzw. 1. Oktober 1946 ein in der Geschichte bis dahin einmaliges Gerichtsverfahren, genauso einmalig wie die unvorstellbaren Verbrechen, die den Angeklagten vorgeworfen und wegen derer sie verurteilt wurden. Noch nie war Führer_innen eines besiegten Landes nach Kriegsende ein regulärer Prozess gemacht worden. Ein großer Teil der dem Verfahren zugrundeliegenden Straftatbestände war erst wenige Wochen vor Prozessbeginn im "Londoner Statut" vom 8. August 1945 und seinem Anhang geschaffen worden. Bisher als unverrückbar geltende Rechtsgrundsätze für Strafverfahren, wie das Verbot der Rückwirkung von Straftatbeständen, wurden dabei außer Kraft gesetzt. ${ }^{2}$ In der juristischen Fachwelt kam es zu teilweise heftig geführten Kontroversen über die RechtmäBigkeit des Prozesses. Führende Staatsrechtler_innen wie etwa Hans Kelsen, Professor an der Universität Berkeley und vor seiner Emigration in die USA Schöpfer der noch heute geltenden Österreichischen Bundesverfassung, lehnten den Prozess strikt ab. ${ }^{3}$

In dieser Arbeit geht es zunächst um die Frage, welchen Stellenwert der Holocaust, also der Völkermord an der jüdischen Bevölkerung Europas, in diesem Prozess hatte. Aus verschiedenen Gründen, die in der Folge näher zu betrachten sein werden, leitet sich als eine erste These dieser Arbeit ab, dass der Holocaust zwar nicht eine der zentralen Stellen in den Anklageschriften einnahm, aber im Gegensatz dazu für die Richter ein wesentlicher Bestandteil ihrer Urteile war. ${ }^{4}$

Neben der Bestrafung der Schuldigen war es ein maßgebliches Anliegen der Alliierten, dem besiegten deutschen Volk die Mitverantwortung an den in seinem Namen verübten Verbrechen aufzuzeigen. Voraussetzung für die Übernahme dieser historischen Verantwortung war es, der deutschen Öffentlichkeit die Realität über den Charakter des nationalsozialistischen Regimes vor Augen zu führen. Für die Aufarbeitung der Vergangenheit war die Kenntnis der Taten und Fakten notwendig. Der Nürnberger Prozess sollte daher nicht nur der Bestrafung der führenden Vertreter des nationalsozialistischen Regimes dienen, sondern auch der deutschen Bevölkerung durch die juristische Aufarbeitung des im Dritten Reich begangenen Unrechts das ganze Ausmaß der in ihrem Namen verübten Verbrechen aufzeigen. ${ }^{5}$ Durch den Umstand, dass der Holocaust im Nürnberger Prozess nur eine untergeordnete Rolle spielte, wurde die deutsche Öffentlichkeit mit diesem Verbrechenskomplex allerdings nur am Rande konfrontiert. ${ }^{6}$ Der zweite Teil der Arbeit beschäftigt sich daher mit der Frage, inwieweit die Deutschen durch die im Prozessverlauf erlangten Informationen von ihrer Mitverantwortung überzeugt werden konnten. Diese Fragestellung muss differenziert beantwortet werden. Während der deutschen Nachkriegsöffentlichkeit das Ausmaß der Verbrechen

2 Hans Ehard, Der Nürnberger Prozess gegen die Hauptkriegsverbrecher und das Völkerrecht, in: Süddeutsche Juristen-Zeitung 3 (1948), Heft 7, S. 353-368, hier S. 358-359.

3 Bradley F. Smith, Der Jahrhundertprozess. Die Motive der Richter von Nürnberg. Anatomie einer Urteilsfindung, Frankfurt a. M. 1977, S. 8

4 Smith, Der Jahrhundertprozess, S. 103-104.

$5 \quad$ Heike Krösche, Zwischen Vergangenheitsdiskurs und Wiederaufbau. Die Reaktion der deutschen Öffentlichkeit auf den Nürnberger Prozess gegen die Hauptkriegsverbrecher 1945/46, den Ulmer Einsatzgruppenprozess und den Sommer Prozess 1958, phil. Diss. Oldenburg 2009, S. 9.

$6 \quad$ Krösche, Zwischen Vergangenheitsdiskurs und Wiederaufbau, S. 183. 
und somit auch der Holocaust großteils bewusst war, lehnte ein bedeutender Teil der deutschen Zivilgesellschaft die Verantwortung dafür dennoch ab, so die zweite These. Insoweit blieb also dem Nürnberger Prozess der von den Alliierten gewünschte Erfolg versagt?

\section{Die rechtlichen Rahmenbedingungen des Prozesses}

Der Zweite Weltkrieg war zwar im geschichtlichen Rückblick bereits 1941 mit dem deutschen Scheitern vor Moskau und 1942 mit Stalingrad und El Alamein entschieden, doch die Seite der Alliierten begann sich erst im Sommer 1944 mit der Nachkriegsordnung in Europa zu beschäftigen. ${ }^{8}$ Dazu gehörte natürlich auch die Frage nach der Behandlung der deutschen Kriegsverbrecher_innen und in diesem Zusammenhang vor allem jene des Umgangs mit den Hauptschuldigen. In diesem Punkt waren sich die Alliierten lange Zeit nicht einig. Großbritannien plädierte dafür, die führenden Nationalsozialisten nach Feststellung ihrer Identität ohne weiteres Verfahren hinzurichten. Bei einem Treffen am 15. September 1944 in Quebec zwischen Roosevelt und Churchill gelang es letzterem, den amerikanischen Präsidenten von dieser Vorgangsweise zu überzeugen. Interessanterweise war es die Sowjetunion, die sich diesem Vorschlag widersetzte: Nach einem Treffen mit Stalin unterrichtete Churchill Roosevelt am 22. Oktober 1944, dass die Sowjetunion auf einem Prozess bestünde. Damit war der britische Plan so gut wie gescheitert. ${ }^{9}$

Aber auch in den Vereinigten Staaten gab es kontroverse Auffassungen. Eine Gruppe um Finanzminister Henry Morgenthau vertrat eine ähnlich harte Linie wie die Briten, während das Kriegsministerium unter Henry Stimson für die Aburteilung der führenden Nationalsozialisten in einem Kriegsverbrecherprozess eintrat. ${ }^{10}$ Nach dem plötzlichen Tod Präsident Roosevelts am 12. April 1945 setzte sich unter seinem Nachfolger Harry Truman letztlich Stimsons Position durch. ${ }^{11}$ Am 2. Mai 1945 wurde Robert Jackson, Richter am Obersten Bundesgericht, zum amerikanischen Hauptankläger im Verfahren gegen Verbrecher der Achsenmächte ernannt.12 Am 3. Mai 1945 entschieden die Außenminister der drei Hauptsiegermächte, dass auch Frankreich als Kläger am Prozess teilnehmen sollte. ${ }^{13}$ Am 7. Mai 1945 unterzeichnete Generaloberst Jodl in Reims die Kapitulationsurkunde gegenüber den Westmächten, am 9. Mai folgte die Kapitulation durch Feldmarschall Keitel in Berlin gegenüber der Sowjetunion. Der Krieg in Europa war beendet.

Die Verhandlungen zur Festlegung der rechtlichen Grundlagen für den Prozess gegen die deutschen Hauptkriegsverbrecher begannen am 26. Juni 1945, wobei es sehr

Krösche, Zwischen Vergangenheitsdiskurs und Wiederaufbau, S. 371.

Smith, Der Jahrhundertprozess, S. 32.

Telford Taylor, Die Nürnberger Prozesse. Hintergründe, Analysen und Erkenntnisse aus heutiger Sicht, München 1994, S. 47-48.

10 Taylor, Die Nürnberger Prozesse, S. 50-51.

Ebd., S. 49

Smith, Der Jahrhundertprozess, S. 51.

Taylor, Die Nürnberger Prozesse, S. 58. 
bald zu Differenzen unter den Teilnehmer_innen kam, vor allem zwischen der amerikanischen und der sowjetischen Delegation. ${ }^{14}$ Die Amerikaner_innen wollten nicht nur die Kriegsverbrechen an sich, sondern, typisch für die angloamerikanische Rechtsprechung, vor allem bereits die Vorbereitung eines Angriffskrieges in Form einer Verschwörung oder strafbaren Verabredung pönalisieren. Darüber hinaus sollten nicht nur einzelne Personen, sondern auch bestimmte Organisationen angeklagt werden, sodass bereits die bloße Mitgliedschaft in einer dieser Organisationen strafbar gewesen wäre. ${ }^{15}$ Für die Sowjetunion war vor allem der erste Punkt in dieser Form unannehmbar, da er auch die eigene Rolle bei der Aufteilung Polens und des Angriffes auf Finnland hinterfragt hätte. ${ }^{16}$

Nach langen Verhandlungen, die mehrmals am Rande des Abbruchs standen, einigte man sich auf einen Kompromiss. Im Artikel 6 des "Londoner Statuts" wurden folgende Verbrechen unter Strafe gestellt:

Artikel 6a: Verbrechen gegen den Frieden, d. h. Planung, Vorbereitung, Einleitung oder Durchführung eines Angriffskrieges;

Artikel 6b: Kriegsverbrechen, d. h. Verletzung der Kriegsgesetze oder-gebräuche;

Artikel 6c: Verbrechen gegen die Menschlichkeit, d. h. Mord, Ausrottung, Versklavung, Deportation und ähnliche Handlungen gegen die Zivilbevölkerung vor und während des Krieges. ${ }^{17}$

Um diese Bestimmungen für die Sowjetunion akzeptierbar zu machen, fügte man einen einleitenden Absatz in Artikel 6 ein, nach dem diese Verbrechen nur dann strafbar sein sollten, wenn sie im Interesse der Achsenmächte verübt worden waren. Weitere Artikel des Statuts betrafen die Nichtberücksichtigung der "Act of State Doctrine" (Artikel 7) sowie des Handelns auf Befehl (Artikel 8) als Schuldausschließungsgründe. Nach dem bis dahin geltenden „Kriegsvölkerrecht“ konnten staatliche Hoheitsakte (Acts of State) nicht von Gerichten fremder Staaten geahndet werden, ebenso waren Handlungen auf Befehl einer übergeordneten Stelle nicht strafbar..$^{18}$

Mit der Unterzeichnung des „Londoner Statuts“ am 8. August 1945 waren die rechtlichen Grundlagen für den Nürnberger Kriegsverbrecherprozess gelegt. Auf Basis der drei Straftatbestände des „Londoner Statuts” wurden vier Anklagepunkte formuliert. Es waren das "Gemeinsamer Plan oder Verschwörung" nach Artikel 6a „,Verbrechen gegen den Frieden" ebenfalls nach Artikel 6a, "Kriegsverbrechen“ nach Artikel 6b sowie "Verbrechen gegen die Menschlichkeit" nach Artikel 6c des Statuts. Der Holocaust, der Völkermord an den jüdischen Menschen Europas, fiel unter den vierten Anklagepunkt „Verbrechen gegen die Menschlichkeit" nach Artikel 6c. Nach weiteren Kontroversen

\footnotetext{
14 Taylor, Die Nürnberger Prozesse, S. 80-81.

15 Ebd., S. 53.

16 Ebd., S. 89.

17 Ebd., S. 746-747.

18 Romy Klimke, Die Nürnberger Prozesse: Ein Meilenstein für die Völkerstrafgerichtsbarkeit - Ein Rückblick zum 70. Jahrestag, in: JURA - Juristische Ausbildung 37 (2015), Heft 12, S. 1265-1274, hier S. 1270.
} 
über die Zusammensetzung der Liste der Angeklagten einigte man sich schließlich darauf, insgesamt 24 Mitglieder der nationalsozialistischen Führungselite vor Gericht zu stellen. ${ }^{19}$

\section{Die Behandlung des Holocaust im Prozess}

Aus unserer heutigen Sicht erstaunlicherweise stand der Holocaust nicht im Mittelpunkt des Prozessgeschehens. Auf Vorschlag der Sowjetunion wurde die Anklage aus organisatorischen Gründen auf die vier Siegermächte verteilt. Die Amerikaner_innen übernahmen die Anklage wegen Verschwörung und gegen die Organisationen, Großbritannien klagte wegen Durchführung von Angriffskriegen. Kriegsverbrechen und Verbrechen gegen die Menschlichkeit, soweit sie in Osteuropa verübt worden waren, fielen in die Zuständigkeit der Sowjetunion, für dieselben Verbrechen im Westen war Frankreich zuständig. ${ }^{20}$

Mit ihrem Anklagepunkt dominierten die Amerikaner_innen den Prozessverlauf. Für sie war der Vorwurf der Verschwörung zu einem Angriffskrieg der zentrale Kern des gesamten Verfahrens. Kriegsverbrechen und Verbrechen gegen die Menschlichkeit wurden von ihnen nur insoweit thematisiert, als sie in diesem Zusammenhang von Bedeutung waren. Robert Jackson, der amerikanische Chefankläger, wollte mit dem Nürnberger Prozess nichts weniger als die Grundlagen für ein neues Kriegsvölkerrecht schaffen. ${ }^{21}$ Das Bestreben der Amerikaner_innen, dem Themenkomplex Verschwörung alles unterzuordnen, führte letztlich zur Vernachlässigung des Holocaust. ${ }^{22}$ Nur wenn Zeugen wie die SS-Obergruppenführer Otto Ohlendorf und Erich von dem Bach-Zelewski aussagten, beide Befehlshaber von Sondereinsatzgruppen, die hinter der Ostfront die massenweise Erschießung der jüdischen Bevölkerung durchführten, kam der Holocaust explizit zur Sprache..3 Aber auch die für den Anklagepunkt Kriegsverbrechen und Verbrechen gegen die Menschlichkeit und damit für den Holocaust eigentlich zuständigen Anklagevertreter aus Frankreich und der Sowjetunion räumten diesem keinen zentralen Platz ein.

Der französische Hauptankläger, François de Menthon, eröffnete am 17. Januar 1946 den französischen Teil der Anklage mit einer beeindruckenden Rede. ${ }^{24}$ Sein darin enthaltenes Loblied auf Frankreich veranlasste Telford Taylor noch nachträglich zu der Bemerkung: „Allerdings fürchte ich, daß es ihm damit nicht gelang, aus dem Gedächnis seiner Zuhörer das andere Frankreich zu vertreiben - das Frankreich von Vichy, Petain und Laval." ${ }^{\prime 25}$

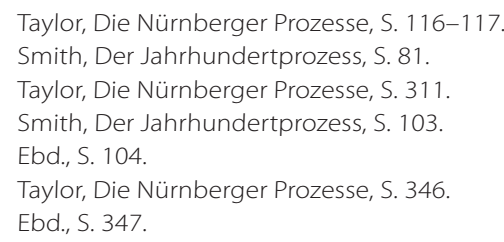


De Menthon war Mitglied der französischen Resistance gewesen und kannte daher die Verhältnisse in Frankreich zur Zeit der deutschen Besatzung aus eigener Erfahrung. ${ }^{26}$ Im Rahmen der französischen Anklage wurden die Kriegsverbrechen in Westeuropa wie Geiselerschießungen, Verschleppung zur Zwangsarbeit, Plünderungen und Zerstörungen sowie die Ausbeutung der französischen Wirtschaft detailliert geschildert, das Schicksal der in Frankreich lebenden jüdischen Menschen allerdings kaum gestreift. Telford Taylor schreibt dazu: „Wenn man die vielen Passagen über Nazi-Kriegsverbrechen in de Menthons Erklärung mehr als vierzig Jahre später nochmals liest, dann stört es einen doch sehr, daß er sich mit keinem Wort auf die Juden und den Holocaust bezieht."27

Für die Französinnen und Franzosen war das Thema Holocaust äußerst unangenehm, hatten doch höchste französische Stellen mit den Deutschen bei der Deportation der jüdischen Bevölkerung in die Vernichtungslager Osteuropas bereitwillig zusammengearbeitet. Das Vichy-Regime hatte eine grundsätzlich antisemitische Einstellung, allerdings ohne die extreme Ausprägung des Antisemitismus des Nationalsozialismus. ${ }^{28}$ Die Kollaboration des Vichy-Regimes erreichte ihren Höhepunkt im Abkommen vom 2. Juli 1942, in dem es sich verpflichtete, die eigene Polizei zur Verhaftung von jüdischen Menschen, die deportiert werden sollten, einzusetzen. Tatsache ist: Ohne die Mithilfe der französischen Polizei wäre den Deutschen eine massenhafte Deportation französischer Jüdinnen und Juden nicht möglich gewesen. ${ }^{29}$ Von 330.000 jüdischen Menschen, die 1940 in Frankreich lebten, wurden 76.000 deportiert und ermordet. ${ }^{30}$ Diese Tatsache spielte für die französischen Ankläger allerdings aus dem genannten Grund so gut wie keine Rolle. So lud die französische Anklage als Zeugin für die in den Konzentrationslagern begangenen Verbrechen mit Marie Claude Vaillant-Couturier ein nichtjüdisches Mitglied der Resistance vor. Vaillant-Couturier war 1942 verhaftet, 1943 nach Auschwitz und 1944 nach Ravensbrück gebracht worden. Sie schilderte ausführlich die entsetzlichen Zustände im Vernichtungslager, von 230 Französinnen, die in ihrem Transport nach Auschwitz kamen, überlebten nur 49.31

Für die sowjetische Anklage stand der Holocaust ebenfalls nicht im Mittelpunkt des Prozesses, wenn auch aus anderen Gründen. Die Sowjetunion wollte die jüdische Bevölkerung nicht als die Hauptopfer des nationalsozialistischen Deutschland behandeln. Für sie standen andere Verbrechen im Vordergrund, vor allem der Massenmord an den sowjetischen Kriegsgefangenen. Die unzureichende Ernährung, Todesmärsche von der Front in die Lager, und die Unterbringung teilweise unter freiem Himmel forderten

\footnotetext{
26 Taylor, Die Nürnberger Prozesse, S. 349.

27 Ebd., S. 349.

28 Serge Klarsfeld, Vichy - Auschwitz. Die Zusammenarbeit der deutschen und französischen Behörden bei der "Endlösung der Judenfrage" in Frankreich (Schriften der Hamburger Stiftung für Sozialgeschichte des 20. Jahrhunderts 9), Nördlingen 1989, S. 322

29 Klarsfeld, Vichy - Auschwitz, S. 325.

30 Ebd., S. 320

31 Taylor, Die Nürnberger Prozesse, S. 355.
} 
eine Unzahl von Opfern. ${ }^{32}$ Von 5.734 .528 sowjetischen Soldaten, die in Gefangenschaft geraten waren, kamen ca. 3.300.000 ums Leben. ${ }^{33}$

Der von den Deutschen in Osteuropa geführte Krieg war, anders als der im Westen, ein Vernichtungskrieg gegen die Bevölkerung mit dem Ziel der Eroberung und Kolonialisierung. ${ }^{34}$ So erklärte SS-Obergruppenführer Erich von dem Bach-Zelewski in seiner Vernehmung vor Gericht am 7. Jänner 1946, Ziel der Deutschen in den eroberten Gebieten sei es gewesen, die slawische und jüdische Bevölkerung um dreißig Millionen zu dezimieren. ${ }^{35} \mathrm{Zu}$ den Exzessen der deutschen Besatzer in den besetzten Ostgebieten sagte er wörtlich: „Wenn man jahrelang predigt, jahrzehntelang predigt, daß die slawische Rasse eine Unterrasse ist, daß die Juden überhaupt keine Menschen sind, dann muß es zu einer solchen Explosion kommen."36

Der sowjetische Hauptankläger war Generalleutnant Roman A. Rudenko, Generalstaatsanwalt der ukrainischen Sowjetrepublik. ${ }^{37} \mathrm{Er}$ begann sein Eröffnungsplädoyer am 8. Februar 1946 und es dauerte immerhin bis zum 25. Februar, bis die Sowjets zum Anklagepunkt "Verbrechen gegen die Menschlichkeit" kamen. ${ }^{38}$ Die Erörterung dieses Punktes nahm nur drei Tage in Anspruch, am 27. Februar 1946 beendeten die sowjetischen Vertreter_innen ihre Anklage. Zwischen dem 8. Februar und dem 25. Februar beschäftigte sich die sowjetische Anklagevertretung vornehmlich mit den Vorbereitungen Deutschlands zum Überfall auf die Sowjetunion, mit den Verbrechen gegen die sowjetischen Kriegsgefangenen sowie der Zivilbevölkerung der Sowjetunion, Jugoslawiens, Polens und der Tschechoslowakei. Darüber hinaus wurden auch die Vernichtung von Kulturgütern und die Plünderungen von Kunstwerken durch die Nationalsozialisten thematisiert. ${ }^{39}$ Erst im Laufe des 26. Februar 1946 geriet der Holocaust in Osteuropa in den Fokus des Prozesses. Es war der Sowjetunion vorbehalten, jüdische Überlebende in den Zeugenstand zu bringen und so den Blick des Prozesses doch noch auf die Verbrechen am jüdischen Volk zu lenken. Es waren das der Schriftsteller Abram Sutzkever, der das Wilnaer Ghetto überlebt hatte, Severina Schmaglewskaja, Häftling in Auschwitz, und Samuel Rajzman, Überlebender des Vernichtungslagers Treblinka, die alle am 27. Februar 1946 aussagten.

Sutzkever berichtete, dass das Wilnaer Ghetto von den Deutschen nur zum Zweck der Vernichtung der jüdischen Bevölkerung der Stadt eingerichtet worden war. ${ }^{40}$ Man

32 Christian Streit, Keine Kameraden. Die Wehrmacht und die sowjetischen Kriegsgefangenen, Stuttgart 1978, S. 137.

33 Streit, Keine Kameraden, S. 244-246.

34 Kershaw, Höllensturz, S. 487.

35 Internationaler Militärgerichtshof Nürnberg (Hrsg.), Der Prozeß gegen die Hauptkriegsverbrecher vor dem Internationalen Militärgerichtshof Nürnberg 14. November 1945 - 1. Oktober 1946, amtlicher Text in deutscher Sprache, Bd. 4, Verhandlungsniederschriften 17. Dezember 1945 - 8. Januar 1946, Nürnberg 1947, S. 539.

36 Internationaler Militärgerichtshof Nürnberg, Der Prozeß gegen die Hauptkriegsverbrecher, Bd. 4, S. 549.

37 Taylor, Die Nürnberger Prozesse, S. 129.

38 Ebd., S. 373.

39 Ebd., S. 363-372.

40 Internationaler Militärgerichtshof Nürnberg (Hrsg.), Der Prozeß gegen die Hauptkriegsverbrecher vor dem Internationalen Militärgerichtshof Nürnberg 14. November 1945 - 1. Oktober 1946, amtlicher Text in deutscher Sprache, Bd. 8, Verhandlungsniederschriften 20. Februar 1946 - 7. März 1946, Nürnberg 1947, S. 336. 
trieb die jüdischen Einwohner_innen Wilnas dort zusammen und erschoss sie. Von ursprünglich 80.000 Jüdinnen und Juden überlebten nur 600 die Vernichtungsaktionen, 79.400 Menschen wurden umgebracht. ${ }^{41}$

Die Zeugin Severina Schmaglewskaja wurde von der sowjetischen Anklagevertretung ausschließlich über das Schicksal der Kinder in Auschwitz befragt. Ihre Aussage brachte besonderes Grauen in den Gerichtssaal. Über das Schicksal der Kinder, die mit den laufenden Transporten in Auschwitz ankamen, berichtete sie:

„Die Frauen aber, die Kinder auf den Armen trugen oder Kinderwagen schoben, und diejenigen, die erwachsene Kinder hatten, wurden zusammen mit diesen Kindern ins Krematorium geschickt. Die Kinder wurden vor den Krematorien von den Eltern getrennt und gesondert in die Gaskammern geführt. Zu der Zeit, als die meisten Juden in Gaskammern vernichtet wurden, wurde ein Befehl erlassen, die Kinder in die Öfen des Krematoriums oder in die Gräben um das Krematorium herum zu werfen, ohne sie vorher zu vergasen." ${ }^{42}$

Auf die Frage des Anklägers, wie das zu verstehen sei, ob die Kinder lebend hineingeworfen worden oder vor der Verbrennung auf andere Weise getötet worden seien, antwortete sie:

„Jawohl, die Kinder wurden lebend in den Graben geworfen. Das Geschrei dieser Kinder konnte man im ganzen Lager hören." ${ }^{43}$

Zu den Kindern, die im Lager geboren worden waren, sagte sie aus, diese seien ihren Müttern sofort nach der Geburt weggenommen worden und verschwunden. Man habe nie feststellen können, was mit diesen Kindern geschehen sei, ob sie noch lebten oder ob sie ermordet wurden: „Im Namen aller Frauen, die im Konzentrationslager zu Müttern geworden sind, möchte ich heute die Deutschen fragen: Wo sind diese Kinder?"44

Als letzter Zeuge zum Holocaust wurde Samuel Rajzman, Überlebender des Vernichtungslagers Treblinka, befragt, der über den Ablauf der Tötungen Auskunft gab. Dazu erklärte er:

"Sofort nach ihrer Ankunft mußten alle Leute innerhalb von 5 Minuten den Zug verlassen und auf dem Bahnsteig Aufstellung nehmen. Sie wurden aus den Zügen gejagt und in Gruppen eingeteilt, Frauen und Kinder zusammen und die Männer abgesondert. Alle mußten sich sofort nackt ausziehen. Dieses Ausziehen geschah unter den Peitschenhieben der deutschen Wache. Die Arbeiter, die dort beschäftigt waren, nahmen sofort die Kleider weg, um sie in die Baracken zu tragen. Die Menschen mußten durch die Straßen bis zur Gaskammer nackt gehen." ${ }^{45}$

\footnotetext{
41 Internationaler Militärgerichtshof Nürnberg, Der Prozeß gegen die Hauptkriegsverbrecher, Bd. 8, S. 340. 
Auf die Frage, wie lange ein Mensch gelebt habe, nachdem er in das Lager Treblinka gekommen sei, antwortete Rajzman:

"Die gesamte Prozedur des Ausziehens und der Weg zur Gaskammer dauerte für Männer 8 bis 10 und für Frauen etwa 15 Minuten. Für die Frauen hat es 15 Minuten gedauert, weil ihnen, bevor sie in die Gaskammer gingen, das Haar abgeschnitten wurde."46

Als ein Beispiel des Sadismus gerade gegenüber Kindern berichtete der Zeuge:

„Aus dem Zug wurde ein zehnjähriges Mädchen mit seiner zweijährigen Schwester gebracht. Als das ältere Mädchen sah, daß ein Mann einen Revolver zog, um die kleine Schwester zu töten, fing es an zu weinen und fragte ihn mit weinender Stimme, warum er die Schwester töten wolle. Er hat die kleine Schwester nicht getötet, sondern sie lebend in den Ofen des Krematoriums geworfen, und dann hat er die ältere Schwester erschossen." ${ }^{\prime 47}$

Zum Schicksal seiner eigenen Familie sagte Rajzman aus:

"Nach zwei Tagen Lagertätigkeit brachte man aus der Stadt Waingrowa meine Mutter, meine Schwester und meine beiden Brüder. Ich musste zusehen, wie diese zur Gaskammer geführt wurden. Einige Tage später, als ich Kleider in die Waggons trug, fanden meine Kollegen die Dokumente meiner Frau, die Photographie meiner Frau und meines Kindes. Das ist alles, was von meiner Familie übrig blieb. Nur eine Photographie." ${ }^{\prime 48}$

Mit der Einvernahme der drei Holocaustüberlebenden war dieser zum Ende der Beweisaufnahme doch noch zum Thema des Prozesses geworden. Von den bis dahin insgesamt siebzig Prozesstagen hatte es der Holocaust allerdings gerade einmal an zwei Tagen in den Mittelpunkt des Verfahrens geschafft.

\section{Prozessverlauf und Urteile}

Mit dem Ende der sowjetischen Anklage folgte am 4. März 1946 die Beweisführung der Verteidigung. Nach den Schlussplädoyers der Verteidiger und Ankläger sowie den Schlussworten der Angeklagten begannen am 1. September die Beratungen des Gerichtshofes über die Urteile. Von den ursprünglich 24 Angeklagten hatte Robert Ley unmittelbar vor Prozessbeginn Selbstmord begangen, Gustav Krupp war schwer krank und verhandlungsunfähig. So wurde der Prozess gegen 22 Personen geführt. Martin Bormann war unauffindbar, gegen ihn wurde in Abwesenheit verhandelt, erst sehr viel später sollte sich herausstellen, dass er zur Zeit des Prozesses bereits tot war. ${ }^{49}$

Alle 22 Beschuldigten waren wegen des Anklagepunktes I, Gemeinsamer Plan oder Verschwörung, 16 wegen des Anklagepunktes II, Verbrechen gegen den Frieden, an-

\footnotetext{
46 Internationaler Militärgerichtshof Nürnberg, Der Prozeß gegen die Hauptkriegsverbrecher, Bd. 8, S. 358.

47 Ebd., S. 359.

48 Ebd., S. 360-361.

49 Annette Weinke, Die Nürnberger Prozesse, München 2006, S. 56.
} 
geklagt. Der Anklagepunkt III, Kriegsverbrechen, betraf 18, der Anklagepunkt IV, Verbrechen gegen die Menschlichkeit, ebenfalls 18 Angeklagte. ${ }^{50}$ Am 30. September 1946 begannen die Richter mit der Verlesung der allgemeinen Urteilsbegründung, am 1. Oktober erfolgte dann die Verkündung der einzelnen Urteile und des Strafmaßes.

Bereits in der allgemeinen Urteilsbegründung wichen die Richter von der amerikanischen Auffassung ab, der Punkt I der Anklage, Gemeinsamer Plan oder Verschwörung, beträfe alle drei weiteren Anklagepunkte. Dazu stellte der Gerichtshof fest, die Verschwörung könne sich lediglich auf die Anklage nach Punkt II, also Verbrechen gegen den Frieden, nicht aber auf die Punkte III und IV, Kriegsverbrechen und Verbrechen gegen die Menschlichkeit beziehen. Eine gegenteilige Rechtsauffassung sei durch das "Londoner" Statut nicht gedeckt.5 ${ }^{51}$ Dies bedeutete eine schwere Niederlage für die amerikanische Anklage und deren Chefankläger Robert Jackson, die ja, wie bereits unter Punkt 3 ausgeführt, ihre Prozessführung auf der zentralen Rolle der Verschwörung aufgebaut hatten und die anderen drei Ankläger lediglich als Zuarbeiter betrachteten. ${ }^{52}$ Dadurch rückten die Anklagepunkte III und IV und damit auch der Holocaust weiter in den Mittelpunkt. Dies zeigte sich am 1. Oktober bei Verlesung der einzelnen Urteile. Von den 22 Beschuldigten, die nach Punkt I angeklagt waren, wurden lediglich acht schuldig, 14 jedoch freigesprochen. Von den 16 nach Punkt II, Verbrechen gegen den Frieden, Angeklagten wurden zwölf verurteilt. Dagegen wurden von den jeweils 18 Angeklagten, denen die Punkte III und IV, also Kriegsverbrechen und Verbrechen gegen die Menschlichkeit vorgeworfen wurden, 16 als schuldig und nur zwei als nicht schuldig erkannt. Insgesamt wurden zwölf Angeklagte zum Tod durch den Strang und sieben zu lebenslänglichen oder langjährigen Haftstrafen verurteilt. Drei Angeklagte wurden freigesprochen. ${ }^{53}$ Von den zum Tod Verurteilten wurden alle zumindest auch wegen Verbrechen gegen die Menschlichkeit verurteilt, der ehemalige Gauleiter von Nürnberg und Herausgeber des antisemitischen Hetzblattes Der Stürmer, Julius Streicher, sogar nur wegen dieses Deliktes. ${ }^{54}$

\section{Die Wahrnehmung in der deutschen Nachkriegsöffentlichkeit}

Um sich ein Bild über die Wahrnehmung des Nürnberger Prozesses in der deutschen Öffentlichkeit machen zu können, muss zunächst die Berichterstattung in den Medien genauer betrachtet werden. Informationen über den Prozess waren für die Deutschen nur über Zeitungen und Rundfunk zugänglich.

Obwohl der Prozess neben der Bestrafung der Hauptschuldigen auch der Aufklärung der deutschen Öffentlichkeit dienen sollte, waren die Plätze im Gerichtssaal für deutsche Berichterstatter_innen sehr limitiert. Bei der Eröffnung des Prozesses war unter den 240 Journalist_innen nur ein einziger Deutscher, der Rundfunkreporter Markus

\footnotetext{
50 Smith, Der Jahrhundertprozess, S. 335.

51 Taylor, Die Nürnberger Prozesse, S. 671-672.

52 Smith, Der Jahrhundertprozess, S. 104.

53 Ebd., S. 335

54 Taylor, Die Nürnberger Prozesse, S. 681.
} 
Wolf aus dem sowjetisch besetzten Teil Deutschlands, ${ }^{55}$ bei der Urteilsverkündung befanden sich unter den 280 Reporter_innen dann vierzig Deutsche. ${ }^{56}$ Dazu kam noch, dass wegen des Papiermangels die Zeitungen nur an zwei bis drei Tagen in der Woche erscheinen konnten und auch nur aus wenigen Seiten bestanden. Die interessierte Bevölkerung musste ihr Bedürfnis nach Informationen daher häufig durch die Lektüre der Aushänge der Zeitungen befriedigen. ${ }^{57}$ So kam der Rundfunkberichterstattung eine nicht unerhebliche Bedeutung zu, obwohl viele Radioapparate, die nach Kriegsende noch vorhanden gewesen waren, von den Alliierten ohne nachvollziehbaren Grund beschlagnahmt wurden. ${ }^{58}$

Über das Interesse der Deutschen am Prozessverlauf gehen die Meinungen auseinander. Eine freie Medienwelt gab es nicht, da alle in Deutschland erscheinenden Zeitungen und Rundfunkmeldungen der Zensur durch die Besatzungsbehörden unterlagen. ${ }^{59}$ So standen die Menschen im Nachkriegsdeutschland den Veröffentlichungen über den Prozessverlauf einerseits misstrauisch gegenüber, andererseits gab es trotz der eingeschränkten Berichterstattung in deutschen Zeitungen ein hohes Bedürfnis nach Informationen. ${ }^{60}$

Umfragen, welche die Amerikaner_innen in ihrer Besatzungszone durchführten, sind zwar sicher kritisch zu hinterfragen, da die Befragten der Besatzungsmacht gegenüber möglicherweise nicht immer ihre wahre Meinung offenlegten, dennoch ist daraus ein gewisses Stimmungsbild ableitbar. So antworteten im November 1945 auf die Frage, ob sie durch den Prozess etwas Neues, ihnen bisher nicht Bekanntes, erfahren hätten, 65 Prozent mit Ja, bis zum Sommer 1946 erhöhte sich dieser Prozentsatz sogar auf 87 Prozent. Von diesen waren es im November 194529 Prozent, im Sommer 1946 sogar 57 Prozent, die erstmals Kenntnis von der Existenz von Konzentrationslagern erhalten haben wollten. Ebenfalls im Sommer 1946 gaben dreißig Prozent an, durch den Prozess vom Völkermord an der jüdischen Bevölkerung Europas erfahren zu haben. ${ }^{61}$

In diesem Zusammenhang stellt sich die Frage, welche Kenntnis die deutsche Bevölkerung während der nationalsozialistischen Herrschaft vom Holocaust tatsächlich hatte. Eine Antwort darauf ist schwierig, da das Wissen der einzelnen Personen sehr unterschiedlich war, sodass man nicht von einem einheitlichen Wissensstand ausgehen kann. ${ }^{62}$ Die systematische Ermordung der jüdischen Bevölkerung Europas unterlag offiziell absoluter Geheimhaltung. ${ }^{63}$ Obwohl von Hitler und anderen hochrangigen Funktionären der NSDAP in Reden immer wieder von der "Vernichtung" und "Ausrottung" der Jüdinnen und Juden gesprochen wurde, vermied die nationalsozialistische

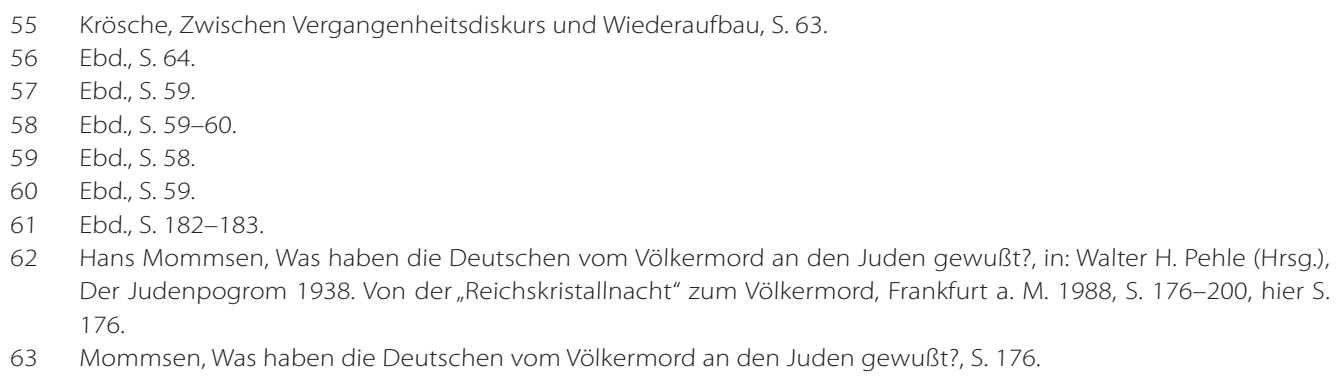


Führung konsequent nähere Angaben über die geplante Vorgangsweise. ${ }^{64}$ Ab 1943 verschwand schließlich jeder Hinweis auf eine „Endlösung" aus der offiziellen Sprachregelung, es wurde nur mehr vom „Arbeitseinsatz in den Ostgebieten" ${ }^{\prime \prime 5}$ gesprochen. Dass aber trotz der verordneten Geheimhaltung Gerüchte über die Ermordung der Jüdinnen und Juden durchsickerten, zeigen die vielen Urteile deutscher Gerichte gegen Menschen, die solche Informationen weitertrugen. ${ }^{66}$ Dabei handelte es sich meist um Meldungen, die die Tätigkeit der Einsatzgruppen hinter der Front betrafen, die Weitergabe von Wissen über Vernichtungslager ist kaum belegbar. ${ }^{67}$ Trotzdem gab es nach heutigem Forschungsstand nicht wenige Menschen, die von Vernichtungslagern mehr oder weniger gesicherte Kenntnis hatten. So kamen in den dem Vernichtungslager Auschwitz angeschlossenen Arbeitslagern die Häftlinge, die ja Wissen über die Vorgänge im Lager hatten, mit den deutschen Beschäftigten der dort tätigen Firmen in Berührung, ebenso gab es sicher zahlreiche Verwaltungsbeamt_innen, die auf Grund ihrer Tätigkeit von der Massenvernichtung wussten. Die Vernichtungslager lagen unmittelbar an Bahnstrecken, Bedienstete der Eisenbahn mussten daher genauso wie Reisende entsprechende Informationen erlangt haben. ${ }^{68}$

Zwischen 1961 und 1998 wurden in Deutschland Umfragen zum Thema Holocaust durchgeführt, deren Aussagewert allerdings mit Vorsicht zu beurteilen ist, da sie durch Vergessen, Verdrängen oder einfach durch Überlagerung mit neuerworbenem Wissen verfälscht sein können. Trotzdem ergab sich daraus durchgängig, dass zwischen 32 und 40 Prozent der Befragten angaben, bereits vor Kriegsende Informationen über den Massenmord an der jüdischen Bevölkerung gehabt zu haben. Dabei wurden aber keine Angaben erhoben, was die Menschen konkret gewusst und inwieweit sie diesen Informationen geglaubt haben.69 Aber auch unter Berücksichtigung dieser Unsicherheiten kann davon ausgegangen werden, dass ein zumindest teilweises Wissen über den Holocaust in weiten Teilen der Bevölkerung vorhanden war. Es war vielleicht nicht die Mehrheit, die vom Massenmord an ihren jüdischen Mitmenschen wusste, aber auch keine kleine, vernachlässigbare Minderheit. ${ }^{70}$

Grundsätzlich wurde der Nürnberger Prozess von den Deutschen während und unmittelbar nach dem Verfahren nicht in Frage gestellt, die Verurteilung der Spitzen des nationalsozialistischen Regimes stempelte diese zu Sündenböcken und so glaubte sich die breite Masse der Bevölkerung von der Schuld entlastet. ${ }^{71}$ Diese Einstellung zum Prozess änderte sich allerdings in den Folgejahren. Waren 1946 noch 78 Prozent der befragten Deutschen der Meinung gewesen, der Prozess sei fair verlaufen, so wurde diese Ansicht 1950 nur mehr von 38 Prozent vertreten. ${ }^{72}$

64 Peter Longerich, „Davon haben wir nichts gewusst!“ Die Deutschen und die Judenverfolgung 1933 -1945, München 2006, S. 201-202.

65 Mommsen, Was haben die Deutschen vom Völkermord an den Juden gewußt?, S. 185.

66 Longerich, „Davon haben wir nichts gewusst!", S. 223.

67 Mommsen, Was haben die Deutschen vom Völkermord an den Juden gewußt?, S. 185.

68 Longerich, „Davon haben wir nichts gewusst!", S. 238.

69 Ebd., S. 239.

70 Ebd., S. 240.

71 Krösche, Zwischen Vergangenheitsdiskurs und Wiederaufbau, S. 185-186.

72 Weinke, Die Nürnberger Prozesse, S. 99. 
In den unmittelbaren Nachkriegsjahren standen für die Deutschen andere Dinge als die Aufarbeitung ihrer Vergangenheit im Vordergrund. Die Nahrungsmittelknappheit, fehlender Wohnraum und Arbeitslosigkeit stellten die Hauptprobleme der Bevölkerung dar. Vor allem im Hungerwinter 1946/47 ging es für viele Menschen ums nackte Überleben. ${ }^{73}$

Der Versuch der Siegermächte, Deutschland zu „entnazifizieren“, nicht nur die führenden Vertreter des Nationalsozialismus vor Gericht zu stellen, sondern auch die vielen mehr oder weniger "kleinen Räder" des Regimes aus den wichtigen Positionen in Gesellschaft und Verwaltung zu entfernen, schlug gründlich fehl. Nicht weniger als acht Millionen Deutsche waren Mitglieder der NSDAP gewesen, hier eine Unterscheidung zwischen Täter_innen und Mitläufer_innen zu treffen, überstieg schlicht die Ressourcen der Alliierten. Dazu kam, dass ein Wiederaufbau Deutschlands ohne die vielen tausenden Personen aus Verwaltung, Justiz, Wirtschaft und Wissenschaft, die nationalsozialistisch vorbelastet waren, nicht möglich gewesen wäre, wobei es gerade im Bereich der Wissenschaft verabsäumt wurde, Vertriebene zurückzuholen, was sich in der Vermittlung von Lehrinhalten auswirkte. So kehrten viele ehemalige Nationalsozialisten, darunter auch schwer Belastete, auf ihre Positionen in der öffentlichen Verwaltung, im Wirtschaftsleben und in der Wissenschaft zurück. ${ }^{74}$ Als Beispiel sei hier Hans Globke genannt, Mitautor und Kommentator der Nürnberger Rassegesetze, der es in den 1950er-Jahren zu einem der einflussreichsten Berater Konrad Adenauers und zum Staatssekretär im Bundeskanzleramt brachte.

Der einsetzende Ost-Westkonflikt und die Gründung der beiden deutschen Staaten 1949 führte zu unterschiedlichen Formen der Aufarbeitung der nationalsozialistischen Vergangenheit. Die DDR leitete ihre Staatlichkeit aus der Revolution 1918/19 ab und legitimierte daraus die sozialistische Gesellschaftsordnung. Die Haltung zum nationalsozialistischen Regime wurde durch die Regierung vorgegeben, eine öffentliche Diskussion darüber gab es daher nicht. Demgegenüber betrachtete sich die Bundesrepublik als Rechtsnachfolgerin des Dritten Reiches. ${ }^{75}$ Doch auch hier führte die Konsolidierung der wirtschaftlichen und politischen Verhältnisse zunächst nicht zu einem gesteigerten Bedürfnis der Öffentlichkeit, sich mit den Verbrechen des Nationalsozialismus näher auseinanderzusetzen. Im Mittelpunkt des Lebens im Westdeutschland der 1950er-Jahre stand der Wiederaufbau, das deutsche „Wirtschaftswunder". Für die Beschäftigung mit der Vergangenheit gab es in dieser Gesellschaft keinen Platz, das Wirtschaftswunder überlagerte die Erinnerung an das Dritte Reich. ${ }^{76}$ So kam es, dass Prozesse im Zusammenhang mit nationalsozialistischen Verbrechen immer seltener wurden. Waren vor deutschen Gerichten in den Jahren 1947 und 1948 noch 4.135 bzw. 4.160 Verfahren eröffnet worden, so waren es in den Jahren 1953 und 1954 nur mehr 226 bzw. 162 Verfahren. Erst in den Jahren 1959 und 1960 stiegen diese Zahlen wieder

\footnotetext{
73 Krösche, Zwischen Vergangenheitsdiskurs und Wiederaufbau, S. 48-49.

74 Kershaw, Höllensturz, S. 652-653.

75 Krösche, Zwischen Vergangenheitsdiskurs und Wiederaufbau, S. 208.

76 Ebd., S. 210
} 
auf 1.075 bzw. 1.326 eingeleitete Verfahren. ${ }^{77}$ Das war unter anderem auf die 1958 erfolgte Einrichtung der Zentralen Stelle der Landesjustizverwaltungen zur Aufklärung von NS-Verbrechen in Ludwigsburg zurückzuführen. ${ }^{78}$

Im Ulmer Einsatzgruppenprozess von 1958 stand der Holocaust erstmals im Mittelpunkt eines Verfahrens vor einem deutschen Gericht. Der vor dem Ulmer Schwurgericht durchgeführte Prozess gegen Verantwortliche für die Ermordung tausender jüdischer Menschen in Litauen bedeutete daher einen Wendepunkt in der judiziellen Aufarbeitung des Holocaust in Deutschland. ${ }^{79}$ Unmittelbar nach dem Ende des Prozesses wurde die erwähnte "Zentrale Stelle" gegründet, die erstmals die systematische Verfahrenseinleitung von Amts wegen erlauben sollte, da bis dahin die Strafverfolgung meist nur auf Grund privater Anzeigen erfolgt war. ${ }^{80}$ Einen ersten Höhepunkt der dadurch in Gang gesetzten Untersuchungen der deutschen Justiz zum Holocaust stellten dann die drei Frankfurter Auschwitz-Prozesse 1963 bis 1968 dar, in denen insgesamt 25 ehemalige Aufseher des Vernichtungslagers Auschwitz zu langjährigen Haftstrafen, davon neun zu lebenslänglich verurteilt wurden.

\section{Schluss}

Mit der Verkündung der Urteile am 1. Oktober 1946 ging der Nürnberger Prozess gegen die Hauptkriegsverbrecher zu Ende. In den frühen Morgenstunden des 15. Oktober 1946 wurden zehn der zwölf zum Tode Verurteilten gehängt. Gegen Bormann war in Abwesenheit verhandelt worden, Göring hatte wenige Stunden vorher Selbstmord begangen.

Die Richter des Nürnberger Gerichtshofes legten die im „Londoner Statut“ geschaffenen Rechtsgrundsätze umsichtig und wohlüberlegt aus. Dadurch, dass der Gerichtshof die Intentionen der amerikanischen Anklage betreffend Verschwörung und gemeinsamer Plan weitgehend verwarf, reduzierte er das Verschulden auf das einzelne Individuum. ${ }^{81}$ Hans Ehard, Untersuchungsführer und Anklagevertreter im Hochverratsprozess 1924 gegen Adolf Hitler und ab 1946 mehrfach bayerischer Justizminister und Ministerpräsident, stellte bereits 1948 in einem Artikel für die Süddeutsche Juristenzeitung die berechtigte Frage, inwieweit die Konzentration der Anklage auf den neu geschaffenen Tatbestand des Angriffskrieges überhaupt notwendig war, da die Angeklagten schon nach den bestehenden Rechtsnormen aller zivilisierten Staaten entsprechend zu bestrafen gewesen wären. ${ }^{82}$

Der Holocaust spielte trotz des unfassbaren Ausmaßes des Völkermordes an der jüdischen Bevölkerung Europas im Nürnberger Prozess nur eine Nebenrolle. Durch die Verurteilung von 16 der 18 wegen Verbrechen gegen die Menschlichkeit Angeklagten,

77 Andreas Eichmüller, Die Strafverfolgung von NS-Verbrechen durch westdeutsche Justizbehörden seit 1945. Eine Zahlenbilanz, in: Vierteljahreshefte für Zeitgeschichte 56 (2008), Heft 4, S. 621-640, hier S. 626.

78 Eichmüller, Die Strafverfolgung von NS-Verbrechen durch westdeutsche Justizbehörden seit 1945, S. 627.

79 Krösche, Zwischen Vergangenheitsdiskurs und Wiederaufbau, S. 219.

80 Ebd., S. 228.

81 Smith, Der Jahrhundertprozess, S. 330.

82 Ehard, Der Nürnberger Prozess gegen die Hauptkriegsverbrecher, S. 364. 
davon zwölf zum Tod durch den Strang, räumte der Gerichtshof dem Holocaust in seinen Urteilen den ihm gebührenden Platz ein.

Der Versuch, die deutsche Bevölkerung durch den Nürnberger Prozess über die Verbrechen des Nationalsozialismus aufzuklären und vor allem auch für die eigene Mitverantwortung zu sensibilisieren, muss allerdings, was zweiteres betrifft, als gescheitert angesehen werden. Bereits im Januar 1946 verfolgten nach einer Umfrage der amerikanischen Besatzungsbehörde zwar 78 Prozent der Bevölkerung die Berichterstattung über den Nürnberger Prozess, trotzdem stimmten weniger als dreißig Prozent der Befragten der Frage nach der Schuld aller Deutschen zu. Auch Versuche, die Deutschen von ihrer Mitschuld durch Aktionen, bei denen etwa auf Plakaten unter der Überschrift „Eure Schuld" grauenhafte Bilder aus Konzentrationslagern gezeigt wurden, zu überzeugen, brachten in dieser Hinsicht wenig Erfolg..$^{83}$ In den 1950er-Jahren beherrschten Verdrängen und Verharmlosen die deutsche Öffentlichkeit. Man flüchtete sich in die alles beherrschende Welt des Wiederaufbaus und des Wirtschaftswunders und konnte so die Beschäftigung mit den Verbrechen des Nationalsozialismus weitgehend vermeiden. Im Mittelpunkt der Erinnerung standen die Kriegsereignisse und nicht das Grauen der Vernichtungslager. Es sollte fast zwanzig Jahre dauern, bis nach dem Heranwachsen einer neuen Generation langsam ein entsprechendes Umdenken Platz griff.

\section{Literatur}

Ehard, Hans, Der Nürnberger Prozess gegen die Hauptkriegsverbrecher und das Völkerrecht, in: Süddeutsche Juristen-Zeitung 3 (1948), Heft 7, S. 353-368.

Eichmüller, Andreas, Die Strafverfolgung von NS-Verbrechen durch westdeutsche Justizbehörden seit 1945. Eine Zahlenbilanz, in: Vierteljahreshefte für Zeitgeschichte 56 (2008), Heft 4, S. 621-640.

Internationaler Militärgerichtshof Nürnberg (Hrsg.), Der Prozeß gegen die Hauptkriegsverbrecher vor dem Internationalen Militärgerichtshof Nürnberg 14. November 1945 1. Oktober 1946, amtlicher Text in deutscher Sprache, Bd. 4, Verhandlungsniederschriften 17. Dezember 1945 - 8. Januar 1946, Nürnberg 1947.

Internationaler Militärgerichtshof Nürnberg (Hrsg.), Der Prozeß gegen die Hauptkriegsverbrecher vor dem Internationalen Militärgerichtshof Nürnberg 14. November 1945 1. Oktober 1946, amtlicher Text in deutscher Sprache, Bd. 8, Verhandlungsniederschriften 20. Februar 1946 - 7. März 1946, Nürnberg 1947.

Kershaw, lan, Höllensturz, Europa 1914 bis 1949, München 2016.

Klarsfeld, Serge, Vichy - Auschwitz. Die Zusammenarbeit der deutschen und französischen Behörden bei der "Endlösung der Judenfrage" in: Frankreich (Schriften der Hamburger Stiftung für Sozialgeschichte des 20. Jahrhunderts 9), Nördlingen 1989. 
Klimke, Romy, Die Nürnberger Prozesse: Ein Meilenstein für die Völkerstrafgerichtsbarkeit - Ein Rückblick zum 70. Jahrestag, in: JURA-Juristische Ausbildung 37 (2015), Heft 12, S. 1265-1274.

Krösche, Heike, Zwischen Vergangenheitsdiskurs und Wiederaufbau. Die Reaktion der deutschen Öffentlichkeit auf den Nürnberger Prozess gegen die Hauptkriegsverbrecher 1945/46, den Ulmer Einsatzgruppenprozess und den Sommer Prozess 1958, phil. Diss. Oldenburg 2009.

Longerich, Peter, "Davon haben wir nichts gewusst!“ Die Deutschen und die Judenverfolgung 1933 - 1945, München 2006.

Mommsen, Hans, Was haben die Deutschen vom Völkermord an den Juden gewußt?, in: Pehle, Walter H. (Hrsg.), Der Judenpogrom 1938. Von der „Reichskristallnacht" zum Völkermord, Frankfurt a. M. 1998, S. 176-200.

Smith, Bradley F., Der Jahrhundertprozess. Die Motive der Richter von Nürnberg. Anatomie einer Urteilsfindung, Frankfurt a. M. 1977.

Streit, Christian, Keine Kameraden. Die Wehrmacht und die sowjetischen Kriegsgefangenen 1941 - 1945, Stuttgart 1978.

Taylor, Telford, Die Nürnberger Prozesse. Hintergründe, Analysen und Erkenntnisse aus heutiger Sicht, München 1994.

Weinke, Annette, Die Nürnberger Prozesse, München 2006.

Dietmar RiedI ist Student der Geschichtswissenschaften an der Universität Innsbruck. Dietmar.Riedl@student.uibk.ac.at

\section{Zitation dieses Beitrages}

Dietmar Riedl, Die Behandlung des Holocaust im Nürnberger Prozess gegen die Hauptkriegsverbrecher und die Wahrnehmung in der deutschen Nachkriegsöffentlichkeit, in: historia.scribere 10 (2018), S. 143-158, [http://historia.scribere.at], eingesehen 19.6.2018 (=aktuelles Datum).

Creative Commons Licences 3.0 Österreich unter Wahrung der Urheberrechte der Autorlnnen. 